\title{
Management of Thymic Neoplasms: Egyptian NCI Experience
}

\author{
Hala Aziz Shokralla1 ${ }^{*}$, Ahmed Elsayed Fathalla² \\ ${ }^{1}$ Medical Oncology Department, National Cancer Institute, Cairo University, Giza, Egypt \\ ${ }^{2}$ Surgical Oncology Department, National Cancer Institute, Cairo University, Giza, Egypt \\ Email: `halaaziz2001@yahoo.com,drasf1975@hotmail.com
}

How to cite this paper: Shokralla, H.A. and Fathalla, A.E. (2017) Management of Thymic Neoplasms: Egyptian NCI Experience. Journal of Cancer Therapy, 8, 64-72. http://dx.doi.org/10.4236/jct.2017.81006

Received: December 30, 2016

Accepted: January 21, 2017

Published: January 24, 2017

Copyright (c) 2017 by authors and Scientific Research Publishing Inc. This work is licensed under the Creative Commons Attribution International License (CC BY 4.0).

http://creativecommons.org/licenses/by/4.0/

\begin{abstract}
Background: Thymic tumors are rare entity with little information regarding outcomes after therapy with curative intent. They are heterogeneous group of neoplastic lesions with wide spectrum of morphologic appearances. They show different presentations with a variable \& unpredictable evolution ranging from indolent attitude to highly infiltrative and metastasizing one. The optimal treatment includes surgical resection, chemotherapy, and radiotherapy. Objectives: To analyze, to report \& to better understand the clinico-pathologic features, results of treatment, and prognostic factors of these tumors. Materials and Methods: From 2008 to 2014, 13 patients (8 men, 5 women) underwent surgical resection of thymic tumor at a mean age of 47 years. Patient demographics, extent of surgical resection, and outcomes were compiled. Demographic variables, use of chemotherapy or radiotherapy, peri-operative variables, recurrence rates, and long term survival were analyzed retrospectively. The Masaoka stage and tumor diameter were recorded along with other variables that potentially influenced survival such tumor grade, site \& number of metastatic disease. Results: The mean age of our patients was 47 years (range: 21 to 58 years) \& 8 cases $(61.5 \%)$ were men. The distribution of patients according to the Masaoka staging at presentation was, stage I ( 6 cases, $47 \%$ ), stage II ( 3 cases, $23 \%)$, stage III in (1 patient, $7 \%$ ), and stage IV (3 cases, $23 \%$ ). Neoadjuvant chemotherapy was administered to 3 patients (23\%) whose tumors were deemed to be more locally advanced precluding R0 resection from the start \& 2 patients (15\%) received neoadjuvant concomitant chemo-radiotherapy. No patient demonstrated an associated immunologic disorder such as myasthenia gravis. In all patients (13 cases, 100\%) pathologic confirmation of thymic tumor was by CT guided fine needle aspiration/biopsy. Surgical resection was done for all our cases (13 patients, 100\%) through median sternotomy approach in (11 cases, 86\%), cervical approach (1 patient, 7\%) \& transverse sternotomy with clamshell incision in 1 patient (7\%) with a large thymic carcinoma whose postoperative pathology revealed a close margin of resection (R1). Resection of nearby structures was done in our cohort to fulfill R0 resection; pulmonary wedge resection in ( 2 cases, 15\%), pleural resection in (5 cases, 38\%), lobectomy in (1 pa-
\end{abstract}


tient, 7\%) \& unilateral phrenic nerve resection was performed in (2 patients, 15\%). None patients with phrenic nerve injury underwent diaphragmatic plication to improve respiratory insufficiency No superior vena cava resection was done in any of our cases. There were no perioperative deaths, tracheostomy, or postoperative respiratory failure occurred. Complete R0 resection was achieved in 12 cases (92.3\%), and of these patients, only 1 patient $(7.7 \%)$ experienced local recurrence whose primary resection was R1. 4 patients received adjuvant chemotherapy or radiotherapy or both. Mean duration of survival in the entire group was 22.7 months (range: 14 to 36 months). At the last follow-up, (8 patients, $61.5 \%)$ were alive without disease, (1 case, 7.5\%) was alive with disease, and (4 cases, $31 \%$ ) were dead. Conclusions: Thymic tumors are amenable to surgical therapy, with increased use of computed tomography imaging. Patients with early stage disease are being identified more frequently. Complete surgical resection appears to have favorable cure rates in these patients. Patients with locally advanced disease can experience long term survival with a multimodality approach.

\section{Keywords}

Thymus, Tumors, Outcome, NCI, Cairo University

\section{Introduction}

Thymic neoplasms are infrequent tumors; however, they represent the commonest adult anterior mediastinal tumors. These tumors represent a wide variety of tumors, with numerous classification systems. The most recent histological classification clearly classifies them in three main categories: thymomas, thymic carcinomas (TC) and neuroendocrine thymic tumors (NETT). Thymic tumors can occur in all ages, with a peak around 30 - 40 years of age in thymomas with Myasthenia Gravis (MG) and 60 - 70 years of age in those without MG. Men and women are affected with the same frequency [1] [2].

Thymomas are neoplasms of the thymus originating from the gland's epithelial tissue. These rare neoplasms account for $50 \%$ of the anterior mediastinal masses, with an incidence of 0.05 per 100,000 person years. Thymomas can be locally invasive and associated with paraneoplastic syndromes which include myasthenia gravis, hypogammaglobulinemia, and pure RBCs aplasia. Histologically, thymomas are usually classified into 5 main WHO subtypes with Masaoka-Koga staging system being the most widely used system for their staging. Patients with thymomas have an increased (2-fold) risk to develop second malignancies [3] [4] [5].

Thymic carcinomas are relatively uncommon among thymic neoplasms (10\%) but are highly aggressive and associated with poor prognosis. They commonly present with locally advanced disease or with distant metastatic disease, precluding surgery as a therapeutic option. This requires a multimodality treatment approach with a combination of surgery, chemotherapy, and radiation therapy to decrease the chances of recurrence and improve survival [6] [7].

NETT are frequently associated with endocrinopathies (up to 30\%), including Cushing's syndrome, multiple endocrine neoplasia type 1 (MEN-type 1 with tumors of the 
parathyroids, pancreatic islet cells, and pituitary gland), and growth hormone releasing hormone $(\mathrm{GHRH})$ hyper-secretion with acromegaly [8].

\section{Materials \& Methods}

From January 2008 to January 2014, thirteen patients (8 men, 5 women) underwent surgical resection of their thymic tumors in National Cancer Institute-Cairo University. Patient demographics variables, extent of surgical resection, use of chemotherapy or radiotherapy, outcomes, recurrence rates and long-term survival were analyzed. The Masaoka stage and tumor diameter were recorded along with other variables that potentially influenced survival such tumor grade, site \& number of metastatic disease. Follow-up status was obtained from institutional records. Statistical analysis of survival was performed using the Kaplan-Meier and univariable log rank tests.

\section{Results}

\subsection{Patients}

The different pathological types, Masaoka stages and patient sex are described in Table 1.

The patients' characteristics in our cohort are reported in Table 2. The mean age of

Table 1. The different pathological types, Masaoka stages and patient sex.

\begin{tabular}{ccccc}
\hline Masaoka Stage (\%) & Thymoma & Thymic carcinoma & Male & Female \\
\hline I (46) & 6 cases & --- & 4 & 2 \\
II (23) & 2 cases & 1 case & 1 & 2 \\
III (8) & 1 case & --- & 1 & --- \\
IV (23) & ---- & 3 cases & 2 & 1 \\
\hline
\end{tabular}

Table 2. Patients' characteristics in our cohort (13 cases).

\begin{tabular}{ccccccc}
\hline Pt & Age (yrs) & Sex & Tumor Size $(\mathrm{cm})$ & Masaoka staging & Alive & Resection Type \\
\hline 1 & 30 & M & 15 & I & Yes & R0 \\
2 & 47 & F & 8 & I & Yes & R0 \\
3 & 58 & F & 13 & I & Yes & R0 \\
4 & 45 & M & 12 & I & Yes & R0 \\
5 & 31 & M & 10 & II & Yes & R0 \\
6 & 57 & M & 11 & II & Yes & R0 \\
7 & 46 & M & 12 & III & Yes & R0 \\
8 & 21 & F & 16 & IV & No & R0 \\
9 & 47 & M & 5.5 & IV & No & R0 \\
10 & 45 & F & 7 & I & Yes & R0 \\
11 & 48 & F & 4.5 & II & No & R0 \\
12 & 50 & M & 5 & IV & No & R1 \\
13 & 47 & M & 5 & I & Yes & R0 \\
\hline
\end{tabular}

$\mathrm{F}=$ female $\mathrm{M}=$ male . 
our patients was 47 years (range; 21 to 58 years) and Mean tumor size was $9.5 \mathrm{~cm}$ (range; 4.5 to $16 \mathrm{~cm}$ ).

Patients present with dyspnea (8 cases), chest pain ( 7 cases) and cough ( 2 cases). There were no thymic neuroendocrine tumors in our work. No patient demonstrated any associated immunologic disorder such as myasthenia gravis.

In all patients' likely thymic neoplasms at presentation, we proceed for total thymectomy with complete excision of the tumor in resectable cases (10 cases). Resectability is feasible in well defined anterior mediastinal mass, in thymic bed, no adenopathy, negative tumor markers (BHCG, AFP, T3, T4, \&TSH) \&not continuous with thyroid [9].

In contrast, in non resectable cases (3 cases) we did CT guided fine needle aspiration/ biopsy for confirmation of thymic tumors.

\subsection{Management}

Neoadjuvant chemotherapy was administered to three patients (23\%) whose tumors were deemed to be advanced precluding $\mathrm{R} 0$ resection with two of them received neoadjuvant concomitant chemo-radiotherapy. The decision to administer chemotherapy or radiotherapy preoperatively was individualized in each patient and based on the extent of tumor invasion. The chemotherapeutic regimen was platinum-based. Partial response to treatment, based on radiographic imaging was seen in all of them.

Surgical resection was done for all our cases through median sternotomy approach in (11 cases, 86\%), cervical approach (1 patient, 7\%) \& transverse sternotomy with clamshell incision ( 1 patient $7 \%$ ) with large thymic carcinoma whose post-operative pathology revealed a close margin of resection (R1). Resection of nearby structures was done in our cohort to fulfill R0 resection; pulmonary wedge resection in (2 cases, 15\%), pleural resection in (5 cases, 38\%), lobectomy in (1 patient, 7\%) \& unilateral phrenic nerve resection was performed in (2 patients, $15 \%)$. No superior vena cava resection was done in any of our cases (Figure 1 \& Figure 2).

No perioperative deaths occurred nor patients required tracheostomy for postoperative respiratory failure. The two patients who had unilateral phrenic nerve resection as part of their operation none of these patients underwent a diaphragmatic plication early in the postoperative course to improve respiratory insufficiency. Four patients (30\%) received adjuvant chemotherapy, radiotherapy or both.

\subsection{Survival \& Follow-Up}

Mean duration of survival in the entire group was 22.7 months (range; 14 - 36 months). At last follow-up, 8 patients (61.5\%) were alive and free of disease, one patient ( $8 \%$ ) was alive with disease (local recurrence) and four patients were dead (31\%). We failed to report the association between all variables analyzed and survival. Figure 3 and Figure 4 showed the overall survival (OS) and disease free survival (DFS) in our cohort.

\section{Discussion}

Thymic tumors are uncommon masses in anterior mediastinum that preclude surgery as a therapeutic option in most cases. We report only 13 cases in four successive years 
at our institute. Most patients are adults in the fifth and sixth decades, with no sex predilection, although, it may occur at any age and better physiologic condition than those with other thoracic malignancies, often thus enabling thymoma patients to undergo more aggressive surgical and medical therapy when indicated [10].

In our cases we have only three cases $>50$ years (range; 21 - 58 years) and all were PS-I. We had Mean tumor size was $9.5 \mathrm{~cm}$ (range; $4.5-16 \mathrm{~cm}$ ) for the 13 patients.

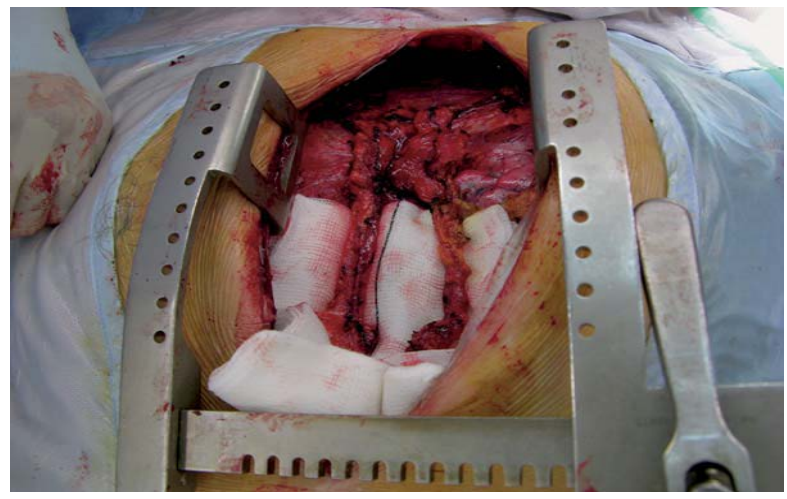

Figure 1. Intraoperative view of dissected thymus with upper pole thymoma.

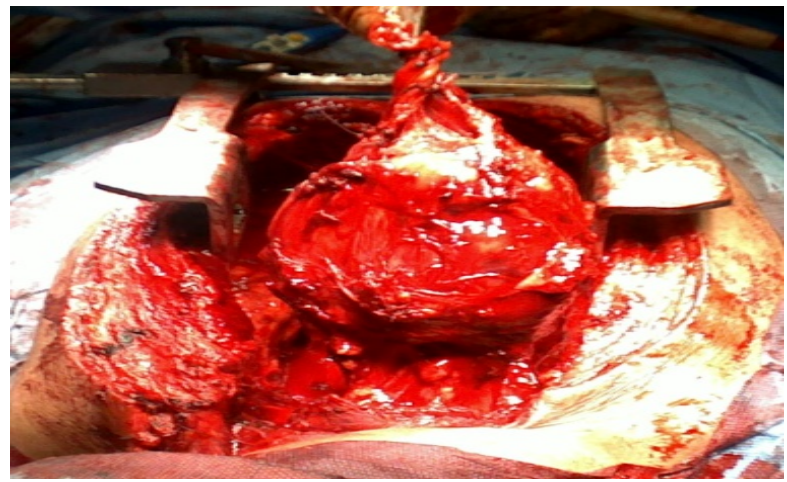

Figure 2. Intraoperative view of large thymic carcinoma.

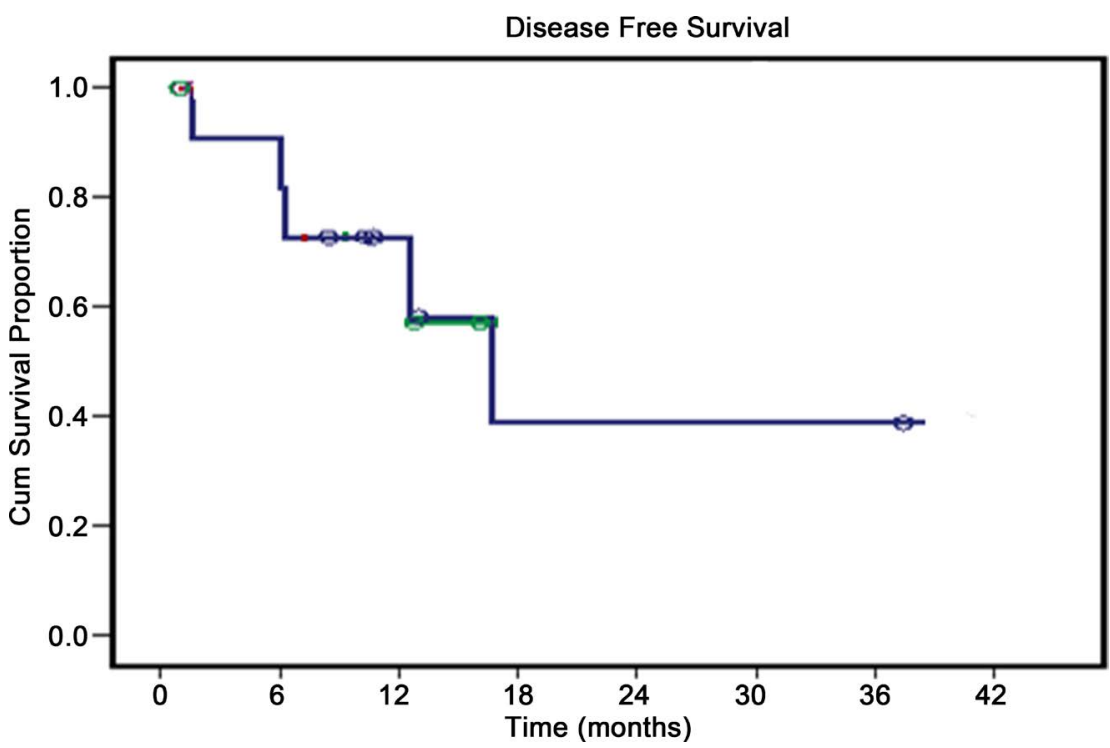

Figure 3. Disease free survival of our cases. 
Overall Survival

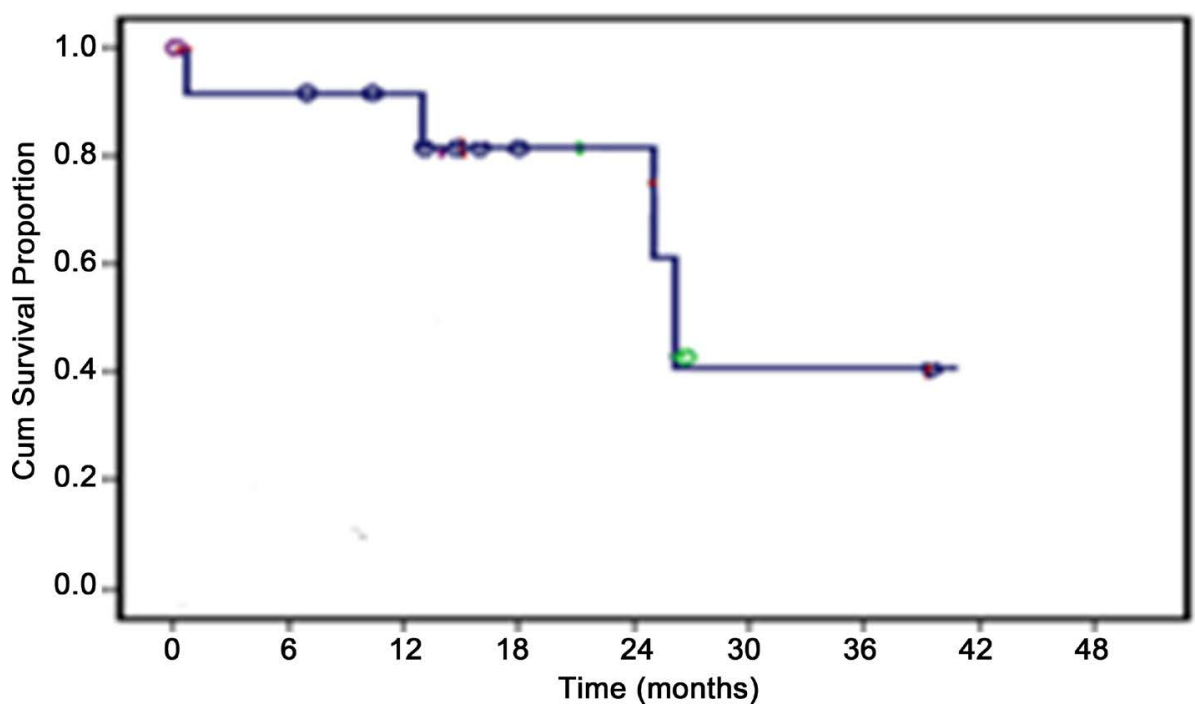

Figure 4. Overall survival of our cases.

Patients may have local symptoms related to the presence of the tumor within the mediastinum. Vague chest pain, shortness of breath and cough are the common complaints. Severe chest pain, superior vena cava obstruction, paralysis of a hemi-diaphragm as a result of involvement of a phrenic nerve, and hoarseness caused by involvement of a recurrent laryngeal nerve are infrequent but are signs of extensive malignant disease. Our Patients present with dyspnea (8 cases), chest pain ( 7 cases) and cough ( 2 cases). There were no thymic neuroendocrine tumors in our work. No patient demonstrated any associated immunologic disorder such as myasthenia gravis [11].

Managing patients with thymic neoplasms is challenging. It is generally agreed that thymoma and thymic carcinoma originate from thymic epithelial cells and that the variable amount of lymphocytes present within the tumor is considered reactive. There have also been multiple attempts to establish a TNM classification which correlated with outcome however, the system most widely accepted is the one proposed by Masaoka et al. in 1981 and modified by Koga in 1994 [12].

As we mention before; we have only four cases of thymic carcinoma.

Our surgical approach to thymic carcinoma is similar to our approach to thymomas, with the intent for complete resection. At our institution, we have avoided surgical "debulking" as part of a treatment plan. All of the patients in our series were felt to have disease potentially amenable to complete resection, and the only patient in our series with incomplete resections had microscopically involved margins only (R1 resection). Surgery is the cornerstone of the management of thymic neoplasms, initially being useful for precise histopathological diagnosis and staging, and in most cases providing the first-line therapeutic modality. For resectable tumors, immediate and complete surgical resection is advocated. During surgery, a careful examination of the mediastinum and pleural cavities, followed by evaluation of macroscopic capsular invasion, peritumoral and pleural adhesions, and involvement of surrounding tissues should be identified and involved in resection. Careful exploration of the mediastinal pleura, especially in the costophrenic angles, may detect droplet metastasis. Areas of uncertain margins 
are marked with clips to allow precise delivery of postoperative RT. Completeness of resection has consistently represented an independent prognostic indicator for thymomas, both in early and advanced disease [13] [14].

Our usual surgical approach included sternotomy, as we believe this approach allows for the best exposure and ability for concomitant pulmonary or great vessel reconstruction. Although involvement of great vessels has been seen as a negative prognostic indicator in other studies, it did not affect postoperative morbidity or overall survival in our series. Furthermore, the extent of pulmonary resection did not influence survival [14] [15].

Complete R0 resection was achieved in 12 cases (92.3\%) and of these patients, only one patient (7.7\%) experienced local recurrence whose primary resection was R1.

The indication of chemotherapy and radiotherapy was individualized for each patient. Previous studies analyzing the effectiveness of chemotherapy have shown mixed results, with response rates of thymic neoplasms to chemotherapy as high as $75 \%$. Our center favored a platinum-based regimen in treating thymic neoplasms, but some centers have reported success with non platinum agents [16].

Our decision to administer neoadjuvant chemotherapy primarily centered on the anticipated ability to resect all disease. If the tumor was felt to be surgically resectable at the diagnosis, then an upfront operation was performed. If the tumor was felt to be invasive, then platinum-based chemotherapy was given first to improve operability potentially. Although neoadjuvant chemotherapy had no statistical effect on overall or disease-free survival, we believe it may have improved our ability to achieve complete resection because all patients who were administered neoadjuvant chemotherapy did have complete resection; we give 3 cases neoadjuvant chemotherapy/radiotherapy [17] [18].

$\mathrm{RT}$ is used in the treatment of thymomas in adjuvant, neoadjuvant, definitive or palliative settings. Post-operative RT is recommended in incompletely resected thymomas. Completely resected stage II and III may also benefit from adjuvant RT to reduce local recurrence rates. Four of our cases received adjuvant therapy (RT/CT or both). Adjuvant chemotherapy has been reported to favorably influence survival in stage III and IVa resected thymomas [19].

The common drugs with good response rates include cisplatin, Adriamycin, etoposide, cyclophosphamide and ifosfamide. Current standard regimens include: Cyclophosphamide, Adriamycin, cisplatin (CAP); cisplatin and etoposide (PE); Adriamycin, cisplatin, vincistine, cyclophosphamide (ADOC); and etoposide, ifosfamide, cisplatin (VIP). Of those whose tumors were completely resected, one patient (7.7\%) experienced a local recurrence.

Chemotherapy is also used as the initial treatment of stage III and IVa unresectable thymomas. The main objective is to reduce the tumor volume to allow subsequent surgery with higher chances of achieving negative margins, or to deliver RT sparing more normal tissues. There is a high-response rates to neoadjuvant CCT, ranging from $77 \%$ to $100 \%$, associated with survival rates ranging from $77 \%$ to $95 \%$. Thus currently, multimodality approach is preferred in locally advanced thymoma, combining all three modalities i.e., chemotherapy, surgery and RT For stage IVb thymomas [18]. In our 
work all stage IV cases received neoadjuvant chemotherapy and RT followed by surgery. Late recurrences occurring more than 5 - 10 years after resection are not uncommon in thymomas. Recurrences are usually locoregional. One patient recurred locally in the present study. Recurrences are managed actively using the three modalitiesSurgery, RT and CCT. Prognostic factors predicting recurrence was evaluated by Detterbeck et al in a systemic review. The significant factors were Masaoka Stage and completeness of resection. Other factors such as age, sex, size of tumor, and PNS were not statistically significant in multivariate analysis [20].

\section{Conclusion}

The overall number of patients with thymic tumors in our series is small (13 cases in 4 years). Therefore, conclusions based on statistical analyses are difficult to achieve. A longer follow-up in all patients may be needed to determine more accurately which factors ultimately are associated with outcome. Additional multicenter studies are needed to determine variables predictive of survival and ideal multimodality and surgical approaches for thymic carcinoma.

\section{References}

[1] Suster, S. and Moran, C.A. (2006) Thymoma Classification: Current Status and Future Trends. American Journal of Clinical Pathology, 125, 542-554. https://doi.org/10.1309/CAV8RNU5TKNACKNC

[2] Engels, E.A. and Pfeiffer, R.M. (2003) Malignant Thymoma in the United States: Demographic Patterns in Incidence and Associations with Subsequent Malignancies. International Journal of Cancer, 105, 546-551. https://doi.org/10.1002/ijc.11099

[3] Rosai, J. and Sobin, L. (1999) Histological Typing of Tumors of the Thymus. In: Rosai, J. and Sobin, L., Eds., World Health Organization, International Classification of Tumors, Springer, Berlin, 9-14. https://doi.org/10.1007/978-3-642-60157-6

[4] Masaoka, A., Monden, Y., Nakahara, K. and Tanioka, T. (1981) Follow-Up Study of Thymomas with Special Reference to Their Clinical Stages. Cancer, 48, 2485-2492. https://doi.org/10.1002/1097-0142(19811201)48:11<2485::AID-CNCR2820481123>3.0.CO; 2-R

[5] Koga, K., Matsuno, Y., Noguchi, M., Mukai, K., Asamura, H., Goya, T., et al. (1994) A Review of 79 Thymomas: Modification of Staging System and Reappraisal of Conventional Division into Invasive and Non-Invasive Thymoma. Pathology International, 44, 359-367. https://doi.org/10.1111/j.1440-1827.1994.tb02936.x

[6] Venuta, F., Anile, M., Diso, D., et al. (2010) Thymoma and Thymic Carcinoma. European Journal Cardio-Thoracic Surgery, 37, 13-25. https://doi.org/10.1016/j.ejcts.2009.05.038

[7] Okereke, I.C., Kesler, K.A., Freeman, R.K., et al. (2012) Thymic Carcinoma: Outcomes after Surgical Resection. The Annals of Thoracic Oncology, 93, 1668-1673.

[8] Ruffini, E., Oliaro, A., Novero, D., et al. (2011) Neuroendocrine Tumors of the Thymus. Thoracic Surgery Clinics, 21, 13-23. https://doi.org/10.1016/j.thorsurg.2010.08.013

[9] www.NCCN.org, 2016.

[10] Tomaszek, S., Wigle, D.A., Keshavjee, S., et al. (2009) Thymomas: Review of Current Clinical Practice. Annals of Thoracic Surgery, 87, 1973-1980. https://doi.org/10.1016/j.athoracsur.2008.12.095

[11] Falkson, C.B., Bezjak, A., Darling, G., Gregg, R., Malthaner, R., Maziak, D.E., et al. (2009) The Management of Thymoma: A Systematic Review and Practice Guideline. Journal of 
Thoracic Oncology, 4, 911-919. https://doi.org/10.1097/JTO.0b013e3181a4b8e0

[12] Detterbeck, F. and Parsons, A. (2004) Thymic Tumors. The Annals of Thoracic Surgery, 77, 1860-1868. https://doi.org/10.1016/j.athoracsur.2003.10.001

[13] Pennathur, A., Qureshi, I., Schuchert, M., et al. (2011) Comparison of Surgical Techniques for Early-Stage Thymoma: Feasibility of Minimally Invasive Thymectomy and Comparison with Open Resection. The Journal of Thoracic and Cardiovascular Surgery, 141, 694-701. https://doi.org/10.1016/j.jtcvs.2010.09.003

[14] Tseng, Y., Wang, S., Wu, M., et al. (2003) Thymic Carcinoma: Involvement of Great Vessels Indicates Poor Prognosis. The Annals of Thoracic Surgery, 76, 1041-1043. https://doi.org/10.1016/S0003-4975(03)00831-2

[15] Kaiser, L.R. (2008) Surgical Treatment of Thymic Epithelial Neoplasms. Hematologyl Oncology Clinics of North America, 22, 475-488. https://doi.org/10.1016/j.hoc.2008.03.009

[16] Lucchi, M., Ambrogi, M.C., Duranti, L., Basolo, F., Fontanini, G., Angeletti, C.A., et al. (2005) Advanced Stage Thymomas and Thymic Carcinomas: Results of Multimodality Treatments. The Annals of Thoracic Surgery, 79, 1840-1844. https://doi.org/10.1016/j.athoracsur.2004.12.047

[17] Yokoi, K., Matsuguma, H., Nakahara, R., Kondo, T., Kamiyama, Y., Mori, K., et al. (2007) Multidisciplinary Treatment for Advanced Invasive Thymoma with Cisplatin, Doxorubicin, and Methylprednisolone. Journal of Thoracic Oncology, 2, 73-78. https://doi.org/10.1097/JTO.0b013e31802bafc8

[18] Koizumi, T., Takabayashi, Y., Yamagishi, S., et al. (2002) Chemotherapy for Advanced Thymic Carcinoma: Clinical Response to Cisplatin, Doxorubicin, Vincristine, and Cyclophosphamide (ADOC Chemotherapy). American Journal of Clinical Oncology, 25, 266268. https://doi.org/10.1097/00000421-200206000-00012

[19] Girard, N. and Mornex, F. (2011) The Role of Radiotherapy in the Management of Thymic Tumors. Thoracic Surgery Clinics, 21, 99-105. https://doi.org/10.1016/j.thorsurg.2010.08.011

[20] Detterbeck, F., Youssef, S., Ruffini, E. and Okumura, M. (2011) A Review of Prognostic Factors in Thymic Malignancies. Journal of Thoracic Oncology, 6, S1698-S1704. https://doi.org/10.1097/JTO.0b013e31821e7b12

\section{Submit or recommend next manuscript to SCIRP and we will provide best service for you:}

Accepting pre-submission inquiries through Email, Facebook, LinkedIn, Twitter, etc. A wide selection of journals (inclusive of 9 subjects, more than 200 journals)

Providing 24-hour high-quality service

User-friendly online submission system

Fair and swift peer-review system

Efficient typesetting and proofreading procedure

Display of the result of downloads and visits, as well as the number of cited articles

Maximum dissemination of your research work

Submit your manuscript at: http://papersubmission.scirp.org/

Or contact jct@scirp.org 